\title{
Long-term Sequela of Intradural Extramedullary Tuberculoma in the Thoracic Dorsal Spinal Cord: Case Report and Review of the Literature
}

\author{
Seil Sohn', Yong Jun $\mathrm{Jin}^{2}$, Ki-Jeong Kim¹, Hyun-Jib Kim ${ }^{1}$ \\ ${ }^{1}$ Department of Neurosurgery, Seoul National University College of Medicine, Bundang Hospital, Seongnam-si, \\ ${ }^{2}$ Department of Neurosurgery, Seoul Paik Hospital, Inje University, Seoul, Korea
}

A 45-year old man, who had tuberculosis five years ago presented with paresthesia, decreased proprioception, and gait disturbance in the lower extremity which were aggravated for a month. Magnetic resonance imaging revealed the T3-7 intradural extramedullary fibrotic mass with dark signal intensity on T2-weighted images. The yellowish material in the thick fibrous mass was confirmed as caseous necrosis. Two days after the operation, the symptoms improved. Although quite rare, intradural extramedullary tuberculoma should be considered as a chronic sequel of the previous medical history of pulmonary tuberculosis or tuberculous meningitis.

Key Words: Intradural extramedullary • Tuberculoma

\section{INTRODUCTION}

Recently, there has been a resurgence of tuberculosis (TB) in developing and developed countries although mostly due to the increasing prevalence of $\mathrm{HIV}^{24)}$. Tuberculosis infection involving the spine may be classified as Pott's spine and Pott's paraplegia, nonosseous spinal tuberculoma (extradural, intradural extramedullary, intramedullary), tuberculous arachnoiditis (myeloradiculopathy), and spinal meningitis ${ }^{6}$. Among these, nonosseous spinal tuberculoma is extremely rare ${ }^{13)}$. From Dastur's review ${ }^{4}$, $64 \%$ of tuberculomas are extradural, $8 \%$ are intramedullary, and $1 \%$ are intradural extramedullary; the rest (27\%) of the lesions involve the arachnoid without dural involvement. Intradural extramedullary tuberculoma of the spinal cord (IETSC) is a rare modality of TB and it is usually associated with the concurrent tuberculous meningitis. Since Compton and Dorsch ${ }^{1)}$ reported an intradural extramedullary tuberculoma case in 1984, there have been 24 cases reported in the English literature ${ }^{13,15,20,22)}$. Even in countries where TB is endemic, intradural extramedullary $\mathrm{TB}$ is rare ${ }^{3)}$. There have been only two cases documented in Korea, ${ }^{8,22)}$. Although the

- Received: September 16, 2011 - Revised: December 18, 2011

- Accepted: January 6, 2012

Corresponding Author: Yong Jun Jin, MD

Department of Neurosurgery, Seoul Paik Hospital, Inje University, 2 Ka Jeo-dong, Jung-gu, Seoul 100-032, Korea

Tel: +82-2-2270-0029, Fax: +82-2-2270-0573

E-mail: jyj0819@gmail.com paradoxical response to antituberculous therapy is suspected of one of the liable pathogenesis, the cause is unclear ${ }^{11)}$. We would like to report a case of intradural extramedullary tuberculoma considered as a long-term sequela.

\section{CASE REPORT}

A 45-year-old man presented with aggravated gait disturbance one month ago affecting his lower extremity. The paresthesia and loss of proprioception were so evident in the lower extremity that he could not stand alone and walk around. Motor weakness in the lower extremity was not definite. Two weeks ago, urinary difficulty developed. Deep tendon reflexes in the knee and ankle joints increased. There was positive on the Romberg test. All of these symptoms are getting worse. However, he did not complain of fever, sweating, and neck stiffness. In the past medical history, the patient suffered from pulmonary tuberculosis five years ago and had been treated with four-drug therapy for nine months. Although he had the mild paresthesia in the lower extremity several months after anti-TB medications at that time, the evaluation of spinal involvement was not performed. The patient has been lived well without any neurologic deficit.

Magnetic resonance (MR) imaging revealed the T3-7 intradural extramedullary plaque-shaped mass with the dark signal intensity on T2-weighted images, isosignal on T1-weighted images, and heterogeneous enhancement on Gadolinium-enhanced images. The lesion had the broader base on the dorsal 
inner dural layer in the thoracic spine and demonstrated the guttering appearance in the direction to the dorsal surface of spinal cord. There was a stalk-like connection between the intradural mass and the most compressed spinal cord (Fig. 1). Computed tomograms (CT) showed the stippled dot of calcification in the stalk-like portion of mass on the T5-6 disc level (Fig. 2). The initial differential diagnoses were given of en plaque
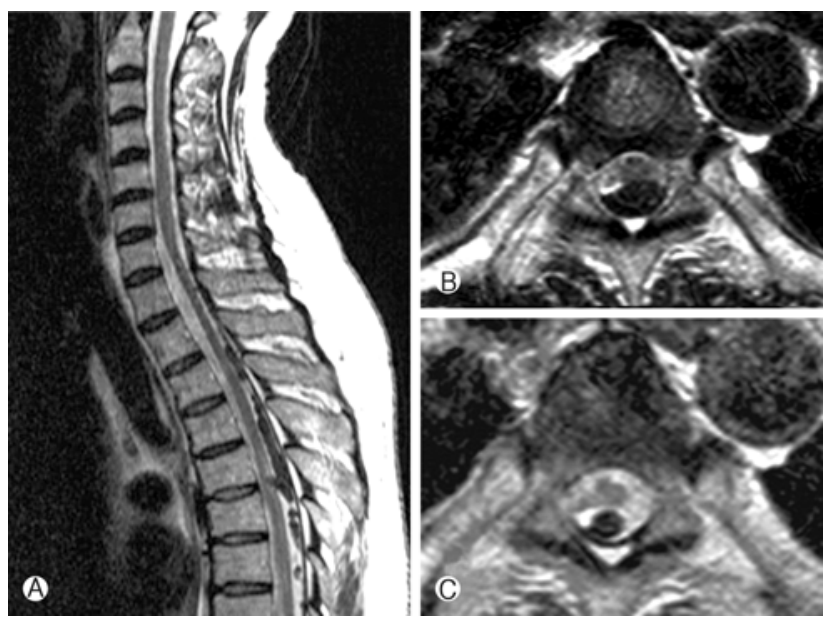

Fig. 1. Magnetic resonance imaging of spine revealed the intradural extramedullary mass with low signal intensity from T3 to T6 vertebral level on the T2-weighted image. The dorsal subarachnoid space was narrow but maintained except connecting stalk between the spinal cord and mass on the T5-6 disc level ( $A$, sagiHal; B, axial section on the stalk level; C, axial).

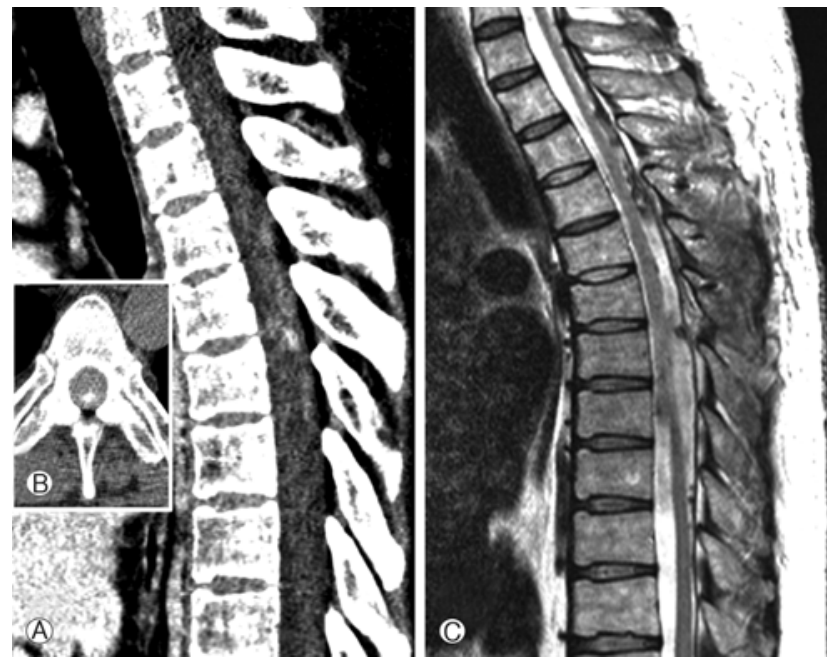

Fig. 2. A and B: Preoperative computed tomography of thoracic spine demonstrating intradural mass with the mottled calcifications in stalk-like connection portion between intradural lesion and spinal cord on the T4-5 disc level. C: Postoperative sagittal T2weighted magnetic resonance image showing the obtaining of dorsal subarachnoid space. meningioma, subdural hematoma with a hidden vascular malformation, and possibly intradural extramedullary tuberculoma. Given the radiologic findings, indolent course of symptom, and the past medical history, a long-term sequela of tuberculoma should be considered as a diagnosis.

The evidence of a progressive pulmonary TB was not found on the X-ray and CT in the chest, which designated old calcified scars in the right upper lung field. Acid-fast bacilli (AFB) of sputum stain and culture were all negative for mycobacterium tuberculosis. The HIV test was also negative. The analysis of cerebrospinal fluid (CSF) did not indicate specific findings (protein 46.3, Glucose 73, RBC 13, WBC 3, poly $0 \%$, lymph 0\%). AFB studies of CSF stain and culture were all negative. Therefore, the lesion was suspected of a chronic inactive fibrotic scar with calcification.

During the operation, T3-7 laminoplastic laminectomies were performed. The dura was found thickened and infiltrated with the underlying mass. The whitish fibrotic mass had the rubberlike consistency. Inside the mass, the yellowish material was scraped out. There was a clear dissection plane between the mass and the spinal cord except in the stalk portion of the mass. The stalk was firmly connected with the pial surface and showed the hard consistency due to calcification. The fibrotic mass was removed with the spinal cord detached completely from the stalk portion of the mass. Somatosensory evo- ked potentials during the operation had been maintaining consistently. However, motor evoked potentials had not been detected during surgery due to technical problems. Pathologic analy-

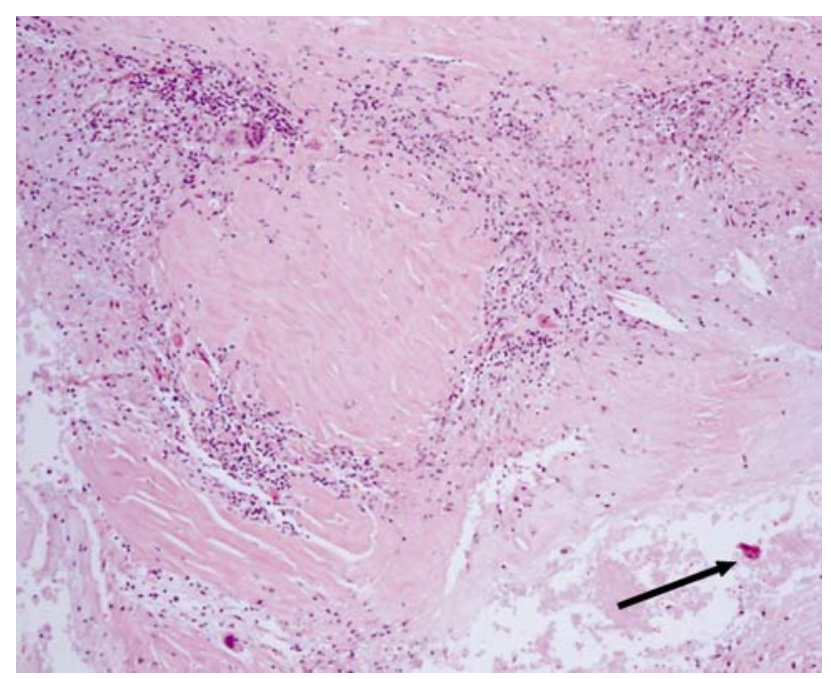

Fig. 3. Photomicrograph showing chronic granulomatous tissue composed of lymphocytes, eosinophils, and multinucleated cells surrounding the fibrotic tissue originated from the previous caseation necrosis. Calcification was seen in the right lower corner (black arrow). (HE stain, $\times 400)$. 
Table 1. Characteristics of previous reported cases of intradural extramedullary tuberculoma after 2000

\begin{tabular}{|c|c|c|c|c|c|c|c|c|}
\hline References & Age & Sex & $\begin{array}{l}\text { Level of } \\
\text { vertebra }\end{array}$ & $\begin{array}{c}\mathrm{Tb} \\
\text { meningitis }\end{array}$ & $\begin{array}{c}\text { Symptom } \\
\text { duration(days) }\end{array}$ & $\begin{array}{l}\text { Time from anti-Tb medication } \\
\text { to surgery (months) }\end{array}$ & Country & Outcome \\
\hline Shim DM et al. ${ }^{22)}$ & 24 & $M$ & T2-6 & Y & 1 & 5 wks & Korea & Good \\
\hline Duan J et el. ${ }^{5)}$ & 14 & $\mathrm{~F}$ & $\mathrm{~T} 1-3$ & Y & 14 & 2 & China & Good \\
\hline Gul $S$ et al. ${ }^{7}$ & 21 & M & $\mathrm{T} 11,12, \mathrm{~L} 1$ & Y & 6 & 3 & Turkey & Good \\
\hline Ozek E et al. ${ }^{19)}$ & 18 & $\mathrm{~F}$ & T1-9 & Y & 7 & 6 & Turkey & Good \\
\hline Takahashi $\mathrm{H}$ et al. ${ }^{24)}$ & 46 & $\mathrm{~F}$ & T 2-3 & Y & 1 & 8 wks & Japan & Good \\
\hline Muthukumar $\mathrm{N}$ et al. ${ }^{16)}$ & 27 & $\mathrm{~F}$ & $\mathrm{~T} 11$ & Y & 60 & 8 & India & Poor \\
\hline Muthukumar $\mathrm{N}$ et al. ${ }^{17)}$ & 21 & M & T6-12 & Y & 90 & 3 & India & Good \\
\hline Luo L et al. ${ }^{13)}$ & 51 & M & T3 & $\mathrm{N}$ & 28 & 4 & USA & Good \\
\hline Kumar $R$ et al. ${ }^{10)}$ & 11 & $\mathrm{~F}$ & T1 1-12 & Y & 60 & 18 & India & Poor \\
\hline Hasan Mirzai ${ }^{15)}$ & 40 & M & C6-T1 & $\mathrm{N}$ & 60 & NA & Turkey & Good \\
\hline Roca B et al. ${ }^{21)}$ & 28 & M & Т3-5 & Y & 30 & 10 wks & Spain & Good \\
\hline Skendros $\mathrm{P}$ et al. $^{23)}$ & 27 & M & $\mathrm{T} 1-4$ & Y & 30 & 3 & Greece & Poor \\
\hline Kim MS et al. ${ }^{8)}$ & 49 & $\mathrm{~F}$ & T1-2 & Y & 1 & 3 & Korea & Good \\
\hline
\end{tabular}

Wks, weeks; Tb, tuberculosis; NA, not available; $Y$, yes; No, no

sis reported the chronic granulomatous inflammation with caseous necrosis, suspicious for tuberculosis (Fig. 3). Pos- toperative MR images showed the most of the granuloma was removed. Two days after the operation, sensory dysfunction and urinary difficulty started to improve. One month after the surgery, he did not complain the gait abnormality and urinary difficulty any more.

\section{DISCUSSION}

Tuberculosis is diagnosed definitely by identifying the causative organism (Mycobacterium tuberculosis) in a clinical sample. When this is not possible, a probable diagnosis may be made using imaging, a tuberculin skin test (Mantoux test), a molecular diagnostic test like polymerase chain reaction (PCR) assays for the detection of bacterial DNA, or a serologic test such as Interferon Gamma Release Assay (IGRA). However, the patient in this report was not confirmed by various diagnostic tests. All the evidences such as the past medial history, results of AFB stain and culture in CSF and sputum, radiological findings, and pathological results suggested that the diagnosis was the chronic sequela of tuberculoma without infectivity.

According to previous reports, intradural extramedullary tuberculomas are usually associated with $\mathrm{TB}$ meningitis ${ }^{12,14)}$. Most cases had history of TB meningitis except four ${ }^{1,2,13,15)}$. This disease seems to also affect young people more commonly and is associated with subacute spinal cord compression with motor and sensory findings that correspond to the level of the lesion. For the review of literatures, searching was limited to the English literatures and was performed using PubMed. References and reviews in identified articles were also examined for potential inclusion. Previous reports before Compton et $\mathrm{al}^{1)}$ were excluded. 24 literatures were included (Table 1). It is interesting to note that all the intradural extramedullary tuberculoma cases except two ${ }^{1,15)}$ were identified after antituberculosis therapy was initiated. The nature of this lesion remains in doubt, but previous reports postulated that the pathophysiology is a result of immunological reaction. Narita et al. ${ }^{18)}$ reported a paradoxical worsening in up to $36 \%$ of patients with tuberculosis and AIDS following an antiretroviral therapy. The mechanism for a paradoxical reaction remains unclear. The most likely explanation is interplay between the host's immune responses and the direct effects of mycobacterial products ${ }^{13)}$. After chemotherapy controls active $\mathrm{TB}$, host-immunosuppression due to TB resolves, and then hypersensitivity to the protein derivatives of mycobacteria causes a paradoxical immune response ${ }^{11)}$. Other possible pathophysiology of calcified tuberculomas was a long-term residual sequel of tuberculosis meningitis ${ }^{9}$. Even though anti- $\mathrm{Tb}$ medication was not initiated, there were two reports with this intradural extramedullary tuberculoma ${ }^{1,15)}$ Although the insidious nature and the gradual progression of the intradural tuberculoma often result in delayed diagnosis ${ }^{6}$, medical therapy still remains the mainstay of the treatment for tuberculomas, while it would seem most reasonable that localized lesions exhibiting pressure symptoms would be treated surgically.

In our case, the patient had symptoms several months after 
anti-TB medications, but he did not undergo further spinal evaluation such as MR imaging at that time because he was followed up in a small public clinic. As his symptoms were mainly insidious sensory symptoms, he could have maintained his work. The cause of progressive thoracic myelopathy occurring five years later was likely to be a mechanical cord compression by a dynamic motion. Despite of the delayed operation, a favorable outcome was obtained. One of the possible explanations of a good outcome is that main symptom was related to the mechanical compression by the old scar tissue without inflammatory changes. This mechanical compression could be relieved by surgical decompression. Another possible cause was that the symptom was not motor weakness but sensory dysfunction indicating the involvement of the dorsal column of spinal cord. Three previous cases ${ }^{10,16,23)}$ of poor outcome were mainly motor symptoms for more than one month. The third possible cause was the presence of clear dissection plane which enabled to be separated from the spinal cord. The adhesion of spinal cord with the tuberculoma was only in the stalk portion. Unfortunately, the severity of adhesion may not be predicted preoperatively using radiological studies.

Given the recent increasing incidence of tuberculosis, the clinician should be aware of its many manifestations, including extrapulmonary disease. Although an intradural extramedullary tuberculoma is a rare entity, it should be included in the differential diagnosis of subacute or chronic spinal cord compression in a relatively young patient with the history of pulmonary $\mathrm{TB}$ or tuberculous meningitis. It is also noticed that a paradoxical reaction may occur after the initiation of antiTB medication. Although medical therapy remains the mainstay of the treatment for tuberculomas, surgical resection will enhance the possibility for a successful outcome if myelopathic symptoms occur.

\section{CONCLUSION}

Intradural extramedullary tuberculoma should be considered in the differential diagnosis of subacute or chronic spinal cord compression in a relatively young patient with the history of pulmonary TB or TB meningitis. A fibrotic mass, which resulted from the long-term sequel of tuberculosis, can be resected. Depending on patient's clinical symptoms and the degree of adhesion to the spinal cord, a favorable outcome can be anticipated.

\section{REFERENCES}

1. Compton JS, Dorsch NW: Intradural extramedullary tuberculoma of the cervical spine. Case report. Journal of Neuro- surgery 60:200-203, 1984

2. Cross JN, Char G, Lowe MV: Intradural extramedullary tuberculoma. The West Indian Medical Journal 35:324-326, 1986

3. Dastur DK, Manghani DK, Udani PM: Pathology and pathogenetic mechanisms in neurotuberculosis. Radiologic Clinics of North America 33:733-752, 1995

4. Dastur HM: Diagnosis and neurosurgical treatment of tuberculous disease of the CNS. Neurosurgical Review 6:111-117, 1983

5. Duan J, Mao B: Unusual intradural extramedullary tuberculoma of the spinal cord in childhood. Neurol India 58:738739, 2010

6. Garg RK: Tuberculosis of the central nervous system. Postgraduate Medical Journal 75:133-140, 1999

7. Gul S, Celebi G, Kalayci M, Acikgoz B: Syringomyelia and intradural extramedullary tuberculoma of the spinal cord as a late complication of tuberculous meningitis. Turkish Neurosurgery 20:561-565, 2010

8. Kim MS, Kim KJ, Chung CK, Kim HJ: Intradural extramedullary tuberculoma of the spinal cord: a case report. Journal of Korean Medical Science 15:368-370, 2000

9. Ku BD, Yoo SD: Extensive meningeal and prenchymal calcified tuberculoma as long-term residual sequelae of tuberculous meningitis. Neurol India 57:521-522, 2009

10. Kumar R, Kasliwal MK, Srivastava R, Sharma BS: Tuberculoma presenting as an intradural extramedullary lesion. Pediatric neurosurgery 43:541-543, 2007

11. Kumar R, Prakash M, Jha S: Paradoxical response to chemotherapy in neurotuberculosis. Pediatric Neurosurgery 42:214222, 2006

12. Lin SK, Wu T, Wai YY: Intramedullary spinal tuberculomas during treatment of tuberculous meningitis. Clinical Neurology and Neurosurgery 96:71-78, 1994

13. Luo L, Pino J: An intradural extramedullary tuberculoma of the spinal cord in a non-HIV-infected patient: case report and review of the literature. Lung 184:187-193, 2006

14. MacDonnell AH, Baird RW, Bronze MS: Intramedullary tuberculomas of the spinal cord: case report and review. Reviews of Infectious Diseases 12:432-439, 1990

15. Mirzai H: Tuberculoma of the cervical spinal canal mimicking en plaque meningioma. Journal of Spinal Disorders \& Techniques 18:197-199, 2005

16. Muthukumar N, Sureshkumar V: Concurrent syringomyelia and intradural extramedullary tuberculoma as late complications of tuberculous meningitis. Journal of clinical neuroscience: Official Journal of the Neurosurgical Society of Australasia 14:1225-1230, 2007

17. Muthukumar N, Sureshkumar V, Ramesh VG: En plaque intradural extramedullary spinal tuberculoma and concurrent intracranial tuberculomas: paradoxical response to antituberculous therapy. Case report. Journal of Neurosurgery Spine 6: 169-173, 2007

18. Narita M, Ashkin D, Hollender ES, Pitchenik AE: Paradoxical worsening of tuberculosis following antiretroviral therapy in patients with AIDS. American Journal of Respiratory and Critical Care Medicine 158:157-161, 1998

19. Ozek E, Iplkcioglu AC, Erdal M: Intradural extramedullary 
tuberculoma mimicking en plaque meningioma. Neurol India 57:211-212, 2009

20. Roca B: Intradural extramedullary tuberculoma of the spinal cord: a review of reported cases. The Journal of Infection 50: 425-431, 2005

21. Roca B, Gonzalez-Darder JM, Lucas A, Borras JM, Pesudo JV: Spinal cord compression from intradural extramedullary tuberculoma. The American Journal of Medicine 117:620-621, 2004

22. Shim DM, Oh SK, Kim TK, Chae SU: Intradural extramedul- lary tuberculoma mimicking en plaque meningioma. Clinics in Orthopedic Surgery 2:260-263, 2010

23. Skendros P, Kamaria F, Kontopoulos V, Tsitouridis I, Sidiropoulos L: Intradural, eextramedullary tuberculoma of the spinal cord as a complication of tuberculous meningitis. Infection 31:115-117, 2003

24. Takahashi H, Ito S, Kojima S, Tanno T, Hattori T: Intradural extramedullary tuberculoma of the thoracic spine: paradoxical response to antituberculous therapy. Intern Med 47:797-798, 2008 